\title{
miR-142-5p Improves Neural Differentiation and Proliferation of Adipose-Derived Stem Cells
}

\author{
Liang Yang $^{\mathrm{a}}$ Zhi-Fei Wang ${ }^{\mathrm{a}} \quad \mathrm{Hao} \mathrm{Wu}^{\mathrm{a}}$ Wei Wang \\ aDepartment of Neurosurgery, the Third Xiangya Hospital, Central South University, Changsha, \\ 'Department of Neurology, the Second Xiangya Hospital, Central South University, Changsha, China
}

\section{Key Words}

miR-142-5p • RhoA $\cdot$ ROCK1 • Adipose-derived stem cells $•$ Neurogenic differentiation

\begin{abstract}
Background/Aims: MiRNAs may regulate neurogenic differentiation of adipose-derived stem cells (ADSCs). In this study, we hypothesized that the miR-142-5p can repress the expression of RhoA/ROCK1 pathway on the neurogenesis of ADSCs. Methods: Deregulated miRNA during neurogenic differentiation of ADSCs were identified. The expression of neuron-specific enolase (NSE) and $\beta$ III tubulin (Neuron-specific class III beta-tubulin) were detected as the markers of neurogenic differentiation by immunostaining and western blot. The targeting of miR-142-5p on RhoA and ROCK1 was verified by dual luciferase assay, qRT-PCR and western blot. The roles of miR-142-5p and the RhoA/ROCK1 signaling pathway were explored by using functional experiments including cell viability and colony formation assays. Results: MiR-142$5 p$ is significantly upregulated during neurogenic differentiation of ADSCs. Knockdown of endogenous miR-142-5p hampered neurogenic differentiation. MiR-142-5p could directly target RhoA and ROCK1 mRNA and repress their expressions, through which it increased the proportion of differentiated cells with positive NSE and $\beta$ III tubulin. RhoA/ROCK1 signaling pathway is involved in miR-142-5p effect on the process of neurogenic differentiation of ADSCs. Conclusion: Our results demonstrate that miR-142-5p functions as a growth promotive miRNA and plays an important role in neurogenic differentiation by targeting RhoA/ROCK1 in ADSCs.
\end{abstract}

\section{Introduction}

Mesenchymal stromal cells (MSCs) can transdifferentiate into osteogenic, chondrogenic, adipogenic, myogenic, fibroblastic, and neuronal lineages, they are considered as a promising source of cellular therapy and regenerative medicine [1,2]. In particular, Adipose-derived stem cells (ADSCs), isolated from adipose tissue, belong to the family of MSCs and can be differentiated into multiple lineages via chemical induction factors [3]. ADSCs share common 
genetic signals with bone marrow MSCs and provides some advantages over other sources of stem cells, such as abundant quantities, minimally invasive procedures for harvest, and autologous origins that will not require immunosuppression in future therapies [4]. ADSCs can differentiate to a variety of specialized cell types. Although differentiation of the ADSCs to neu ronal and glial lineages has been reported previously [5], the mechanisms underlying the neurogenic differentiation of ADSCs remains elusive.

MicroRNAs (miRNAs) are a class of small noncoding RNAs involved in post transcriptional regulation of gene expression usually by binding to the 3 ' untranslated region (UTR) of mRNAs. Some recent studies indicate that multiple miRNAs might regulate neuronal-like differentiation of MSCs. MiR-21 has been confirmed to directly contribute to ADSCs differentiation [6]. MiR-210 increases ADSCs proliferation and migration via PTPN2 expression [7]. MiR-34a inhibits ADSCs differentiation by regulating the cell cycle and senescence induction [8]. The Let-7 family plays as a pre-differentiated factor with antistemness properties [9]. The functions of miR-26a, miR-181a, miR-222 and miR-200c more potentially mimicked retinoic acid on induction of stem cell neuronal differentiation [10]. Thus, miRNAs could control the proliferation and differentiation of ADSCs. In this study, the role of miR-142-5p in rat ADSCs neuronal proliferation and differentiation was explored. Our results showed that miR-142-5p represses the proliferation and differentiation of ADSCs, which may be mediated by targeting RhoA/ROCK1 signaling.

\section{Materials and Methods}

\section{Cell culture and induction of neurogenic differentiation}

This study was approved by the ethic committee of the Central South University. Human ADSCs were purchased from Invitrogene (Carlsbad, CA, USA) and cultured using the prepackaged MesenPRO RS ${ }^{\mathrm{TM}}$ Medium in an incubator with a humidified atmosphere and $5 \% \mathrm{CO}_{2}$ at $37^{\circ} \mathrm{C}$. Passage $3 \mathrm{ADSCs}$ were used for induction of differentiation.

Naive ADSCs cells or transfected ADSCs cells with miR-142-5p, RhoA, or ROCK1 shRNA were seeded in 12 -well plates at a density of $5 \times 10^{4}$ cells $/ \mathrm{cm}^{2}$. At $40 \%$ confluence, the cell culture was supplemented with $5 \mu \mathrm{g} / \mathrm{ml}$ insulin, $0.5 \mu \mathrm{M}$ retinoic acid and $15 \mathrm{ng} / \mathrm{ml}$ EGF for 2 days. Then, the culture medium was changed to the medium supplemented with $100 \mu \mathrm{M}$ cAMP, $500 \mu \mathrm{M}$ 3-isobutyl-1-methylxanthine (IBMX), 100ng/ml nerve growth factor (NGF) and $10 \mu \mathrm{M}$ forskolin for another 3 days.

\section{Immunostaining}

To assess neurogenic differentiation, the cells were stained with neuron-specific enolase (NSE), and Neuron-specific class III beta-tubulin ( $\beta$ III tubulin). Briefly, cells were fixed with $4 \%$ paraformaldehyde for 20 min. Then the cells were permeabilized using PBS containing 0.5\% Triton-X 100 (Sigma Aldrich) for 1 hour and then blocked using PBS supplemented with 4\% BSA and 0.3\% Triton-X 100 for another hour. After these treatments, the cells were incubated primary anti-NSE antibody (1:200, Abcam, Cambridge, MA, USA) or anti- $\beta$ III tubulin $\left(1: 200\right.$, Abcam) overnight at $4^{\circ} \mathrm{C}$, followed by incubation with fluorescein isothiocyanate conjugated goat anti-rabbit antibody (1:5000 dilution, KPL) at room temperature for $60 \mathrm{~min}$. Finally, the nuclei were stained with $5 \mathrm{mg} / \mathrm{ml}$ DAPI for $15 \mathrm{~min}$ and images were acquired using a fluorescence microscope (IX81; Olympus, Tokyo, Japan).

\section{Reagents and cell transfection}

The pLVmiR-142-5p expression plasmid, the pLVmiR-142-5p locker plasmid and the lentiviral packaging vector mix were purchased from Biosettia (San Diego, CA, USA). The lentiviral pLV-miR expression vector or the pLV-miR locker vectors were produced by co-transfection with helper plasmids mixture into HEK 293T cells using FuGene HD (Promega Corporation, Madison, WI) according to manufacturer's instruction. The ready-to-use human RhoA shRNA lentiviral particles and ROCK1 shRNA lentiviral particles were purchased from Santa Cruz Biotech (Santa Cruz, CA, USA). To overexpress or suppress the genes, the ADSCs were infected with corresponding lentiviral particles with the presence of $8 \mu \mathrm{g} / \mathrm{ml}$ polybrene (SigmaAldrich, MO, USA). 


\section{Cellular Physiology Cell Physiol Biochem 2018;50:2097-2107 and Biochemistry \begin{tabular}{l|l} 
DOI: 10.1159/000495054 & (c) 2018 The Author(s). Published by S. Karger AG, Basel \\
www.karger.com/cpb
\end{tabular}}

Yang et al.: miR-142-5p Targeting RhoA/ROCK1 in ADSCs

Taqman miRNAs array

ADSCs before induction, 2 days after induction and 5 days after the induction were used for microarray of miRNAs expression. Briefly, total miRNAs in the cell samples were extracted using the miRVana miRNA Isolation Kit (Ambion, Austin, TX, USA) according to the manufacturer's instructions. Then, TLDAv3.0 was performed as the manufacturer's protocol. All PCR reactions were performed on the 7900HT real-time PCR system (Applied Biosystems). Cycling conditions were as follows: $95^{\circ} \mathrm{C}$ for $10 \mathrm{~min}$ followed by 40 cycles of $95^{\circ} \mathrm{C}$ for $15 \mathrm{~s}$ and $60^{\circ} \mathrm{C}$ for $1 \mathrm{~min}$. U6 snRNA was used as an internal control to normalize RNA input. The data were analyzed by SDS v2.3 software. In details, the Ct value is defined as the fractional cycle number at which the fluorescence passes the fixed threshold. The fold change was calculated using the $2^{-\Delta \Delta} \mathrm{CT}$ method.

qRT-PCR analysis

Total RNAs of cells were extracted using Trizol Reagent (Invitrogen) according to the manufacturer's instruction. Then the RNA samples were used for reverse transcription to get the first strand cDNA by using the PrimeScript1 RT reagent kit (TaKaRa Bio, Dalian, China). The expressions of RhoA and ROCK1 mRNA and neurogenic markers NSE and $\beta$ III tubulin were quantified using gene specific primers with SYBR1 Green PCRMaster Mix (TaKaRa Bio). GAPDH was used as the endogenous control gene. MiR-142-5p expression was detected using TaqMan MicroRNA Assay Kit (Applied Biosystems), with U6 snRNA used as the endogenous control. All qRT-PCR analysis was performed using an ABI Prism 7500 (Applied Biosystems). The results of qRT-PCR analysis were presented using $2^{-\Delta \Delta} \mathrm{CT}$ method.

Cell viability and colony formation assay.

ADSCs were infected with pLV-miR-142-5p locker particles and/or RhoA and ROCK1 shRNA. After 24 $\mathrm{h}$ incubation, cells were placed in a fresh 96-well plate in triplicate for at least 5 days. Cells were tested for proliferation everyday using Cell Titer-Blue cell viability assay (Promega Corporation) according to the manufacturer's instructions and the fluorescence value was recorded by multi-plate reader (Synergy 2; BioTek, Winooski, VT). For CFU assay, transfected cells were placed in a fresh six-well plate in triplicate for 10 days. Then cell colonies were fixed with $20 \%$ methanol and stained with $0.1 \%$ coomassie brilliant blue R250 at room temperature for $15 \mathrm{~min}$. The colonies were counted by ELIspot Bioreader 5000 (BIO-SYS, Karben, GE).

\section{Dual luciferase assay}

Chemically synthesized miR-142-5p mimics and the scramble negative controls were purchased from RiboBio (Guangzhou, China). The fragments of 3'-UTR of human RhoA and ROCK1 containing the putative miR-142-5p binding sequences was amplified with the primers RhoA: 5'- aaccttgctgcaagcacagcccttatgc-3' (forward) and 5'-tgagtgccacccatgagaactggtggctc-3' (reverse) or ROCK1: 5'-ctgagtgccetgtggaatcgtgtggg-3' (forward) and 5'-aatttgaagtatgttttccattcatttct-3' (reverse). The PCR products was cloned into firefly luciferase reporter vector pGL3 (Promega Corporation), termed as pGL3-RhoA-3'UTR and pGL3-ROCK13'UTR. The plasmids, which carried the mutated sequence in the complementary sites for the seed region of miR-142-5p (Fig. 4A) were generated based on pGL3-RhoA-3'UTR and pGL3-ROCK1-3'UTR plasmid by MutanBEST Kit (Takara Bio), termed as pGL3-RhoA-3'UTR mut and pGL3-ROCK1-3'UTR mut. The point mutations along the miR-142-5p seed binding sites were confirmed by sequencing analysis.

To assess the influence of putative miR-142-5p binding site on luciferase expression, HEK 293T cells were co-transfected with 200ng recombinant plasmids and 50nM miR-142-5p mimics or negative control (NC) using FuGene HD. The pRL-TK (Promega Corporation) was also transfected as a normalization control. 48h after transfection, luciferase activity was analyzed using the Dual-Luciferase Reporter Assay System (Promega Corporation) and recorded by multi-plate reader (Synergy 2; BioTek).

\section{Western blot}

Cells were lysed using a RIPA buffer (Beyotime, Shanghai, China) with $0.5 \%$ sodium dodecyl sulfate in the presence of proteinase inhibitor cocktail (Complete mini; Roche). Then the protein concentration was measured using a BCA Protein Assay Kit (Beyotime). Twenty-five micrograms of protein were separated by 10\% SDS-PAGE mini gels and then transferred onto a PVDF membrane (Immobilon P-sQ; Millipore, Billerica, MA). After blocking with 5\% nonfat milk, the membranes were incubated with primary anti-RhoA (1:1000, Abcam), anti-ROCK1 (1:1000,Abcam) or anti-actin (1:5000, Epitomics, Burlingame, CA) at $4^{\circ} \mathrm{C}$ overnight, 


\section{Cellular Physiology Cell Physiol Biochem 2018;50:2097-2107

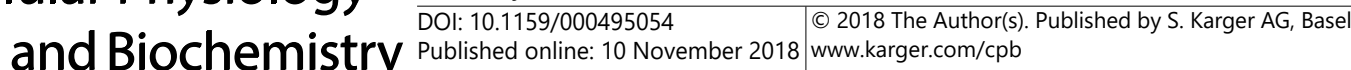 \\ Yang et al.: miR-142-5p Targeting RhoA/ROCK1 in ADSCs}

followed by incubated with HRP-labeled secondary antibodies (anti-rabbit IgG, 1:10000, KPL, Gaithersburg, MA) for $1 \mathrm{~h}$ at room temperature. Finally, the band signals were developed with Super Signal West Pico chemoluminescent substrate (Pierce, Rockford, IL), visualized by the GeneGnome HR Image Capture System (Syngene, Frederick, MD) and analyzed by Gene tools (Syngene).

\section{Prediction of miRNAs targets}

To investigate the target genes of miRNAs and the conserved sites bound by the seed region of miR142-5p, the TargetScan (http://www.targetscan.org/), PicTar (http://pictar.mdc-berlin.de/) and MiRanda (http://www.microrna.org/microrna/home.do) programs were used.

\section{Statistical analysis}

Data were presented in the form of means \pm standard deviation (SD) based at least three repeats of three times independent studies. One-way ANOVA was performed to compare means of in multiple group experiments. Comparison between groups was performed using the unpaired t test. A two-sided P value of $<0.05$ was considered statistically significant.

\section{Results}

miR-142-5p is significantly upregulated during neurogenic differentiation of ADSCs

After the induction, the differentiation was visualized by immunofluorescent staining of neurogenic markers, including NSE and $\beta$ III tubulin (Fig. 1A). By Taqman miRNAs array, a series of miRNAs were found to be significantly desregulated during the induction process. The six most upregulated and six downregulated miRNAs are shown in Fig. 1B. Quantitative RT-PCR (qRT-PCR) analysis confirmed that miR-142-5p expression is consistently increased during the induction (Fig. 1C).

miR-142-5p knockdown inhibits neurogenic differentiation and proliferation of ADSCs

To further verify the effect of miR-142-5p on neurogenic differentiation of ADSCs, the ADSCs were infected with pLV-miR-142-5p locker particles or the vector negative control (NC) (Fig 2A). MiR-142-5p knockdown resulted in substantially decreased expression of NSE and $\beta$ III tubulin mRNA after induction (Fig, 2B). In addition, miR-142-5p knockdown also reduced the proportion of cells with positive NSE $(51.8 \pm 4.7 \%$ vs. $27.4 \pm 3.6 \%)$ and $\beta$ III tubulin $(42.6 \pm 2.5 \%$ vs. $25.6 \pm 3.7 \%)$ after induction (Fig. 2 C), suggesting a repressed differentiation. Moreover, infection with pLV-miR-142-5p locker particles in ADSCs decreased the cell viability (Fig. 3A). Colony-forming unit (CFU) assay also showed that the knockdown of miR142-5p in ADSCs decreased the number of CFU (Fig. 3B).

\section{miR-142-5p directly targets RhoA and ROCK1}

Based on prediction, it was found RhoA and ROCK1 had the putative binding sites with miR-142-5p (Fig. 4A). In addition, miR-142-5p mimics repress the luciferase activity of wild type RhoA and ROCK1 3'UTR sequence, but not the mutant sequence in HEK293T cells (Fig. 4B). In ADSCs, infection with pLV-miR-142-5p expression particles also significantly decreased RhoA and ROCK1 protein expression (Fig. 4C). Moreover, RhoA and ROCK1 expression in the ADSCs during the induction process were significantly downregulated (Fig. 4D). These results suggest that miR-142-5p can directly target RhoA and ROCK1, and regulate their expressions. 


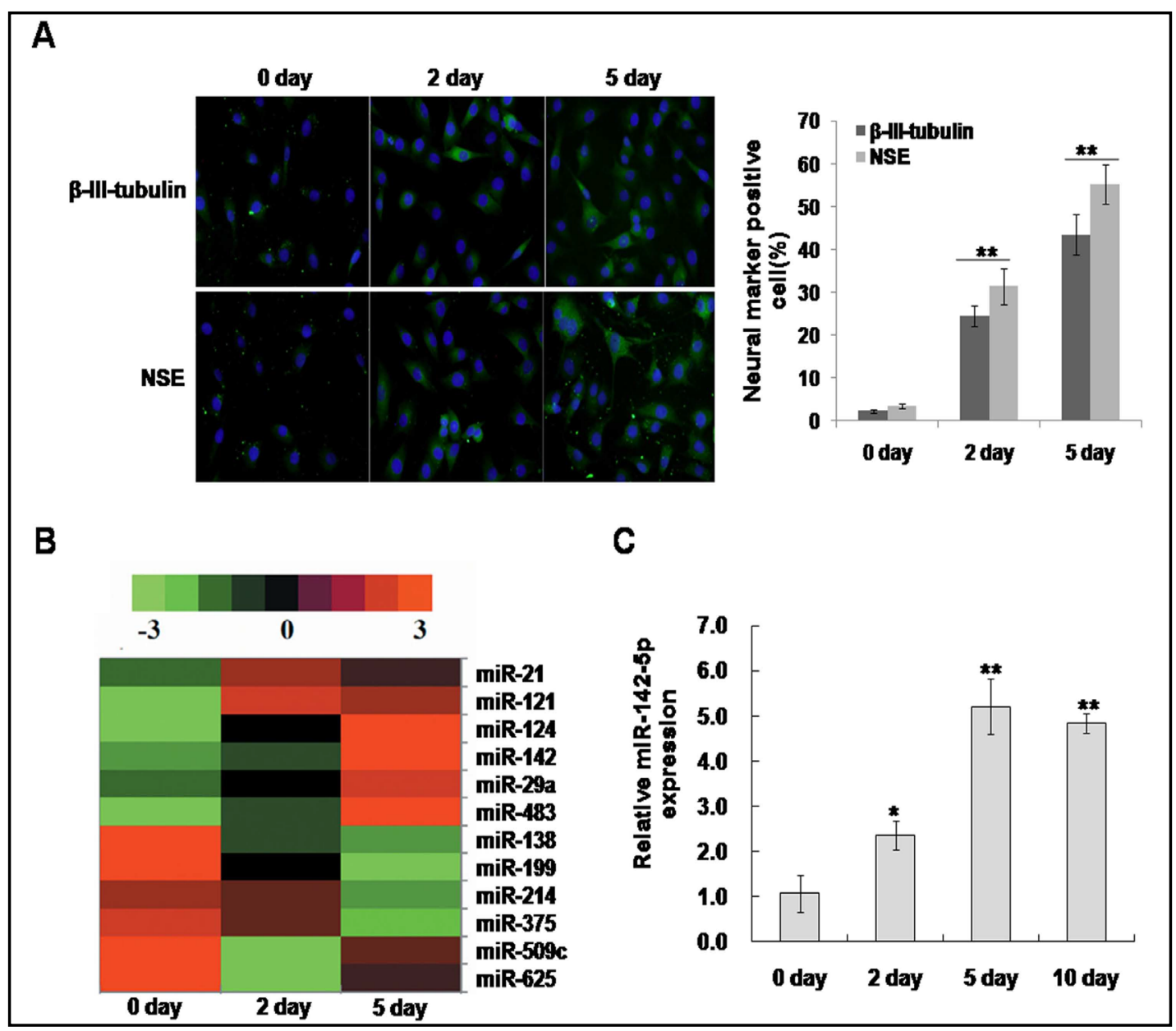

Fig. 1. MiR-142-5p is significantly upregulated during neurogenic differentiation of ADSCs. A. Left panel: representative immunofluorescent images of neuronal markers (NSE and $\beta$ III tubulin) expressed in differentiated ADSCs on day 2 and 5 post-induction. Green: neurogenic markers. Blue: DAPI. Right panel: quantification of the proportion of ADSCs with positive neurogenic markers showed in left panel. B. Twelve most significantly differential expressed miRNAs were identified in the ADSCs on day 0,2 and 5 of the induction. Columns represent samples and rows represent miRNAs (black, green, and red correspond to unchanged, down-regulated and upregulated, respectively). C. qRT-PCR analysis of miR-142-5p expression of the ADSCs on day 0, day 2 and day 5 of induction. Data are shown as the mean \pm S.D. of six independent experiments. Transdiff. $=$ transdifferentiated. ${ }^{*} \mathrm{p}<0.05,{ }^{* *} \mathrm{p}<0.01$.

RhoA/ROCK1 signaling pathway is involved in neurogenic differentiation and proliferation of ADSC by miR-142-5p

To further explore whether RhoA/ROCK signal is involved in miR-142-5p's regulation over neurogenic differentiation and proliferation of ADSCs, ADSCs were transfected with RhoA, ROCK1 or NC shRNA lentiviral particles before induction (Fig. 5A). Knockdown of endogenous RhoA or ROCK1 partly restored the mRNA expressions of neuronal markers (NSE and $\beta$ III tubulin) hampered by miR-142-5p shRNA (Fig. 5B). Immunostaining analysis showed that RhoA and ROCK1shRNA, but not NC shRNA partly reversed the effect of miR142-5p knockdown on repressing the proportion of ADSCs differentiation (Fig. 5C). These results suggest that RhoA or ROCK1 shRNA rescued neurogenic differentiation hampered by miR-142-5p knockdown. CFU assay showed that RhoA and ROCK1shRNA partly reversed the effect of miR-142-5p knockdown on repressing cell proliferation in ADSCs (Fig. 5D). Thus, it is possible that miR-142-5p can modulate neurogenic differentiation and proliferation of ADSCs by simultaneously repressing RhoA and ROCK1.

\section{KARGER}




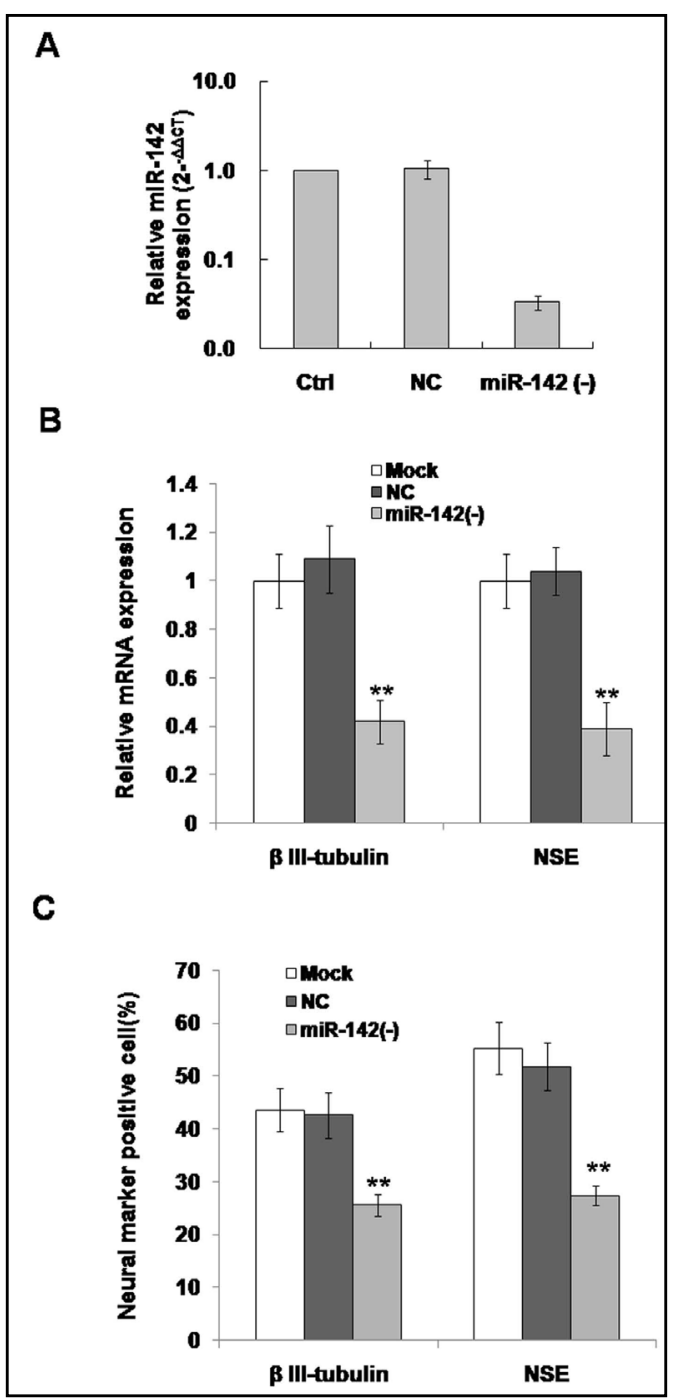

Fig. 2. MiR-142-5p knockdown represses neurogenic differentiation of ADSCs. A. qRT-PCR analysis of miR-142-5p expression in ADSCs infected with the pLV-miR-142-5p locker lentiviral particles or the vector negative control (NC). B. qRT-PCR analysis of NSE and $\beta$ III tubulin mRNA expression in ADSCs on day 5 post-induction. C. Quantification of the proportion of ADSCs with positive neurogenic markers by immunostaining analysis of NSE and $\beta$ III tubulin on day 5 postinduction. Data are shown as the mean \pm S.D. of six independent experiments. ${ }^{* *} \mathrm{p}<0.01$.

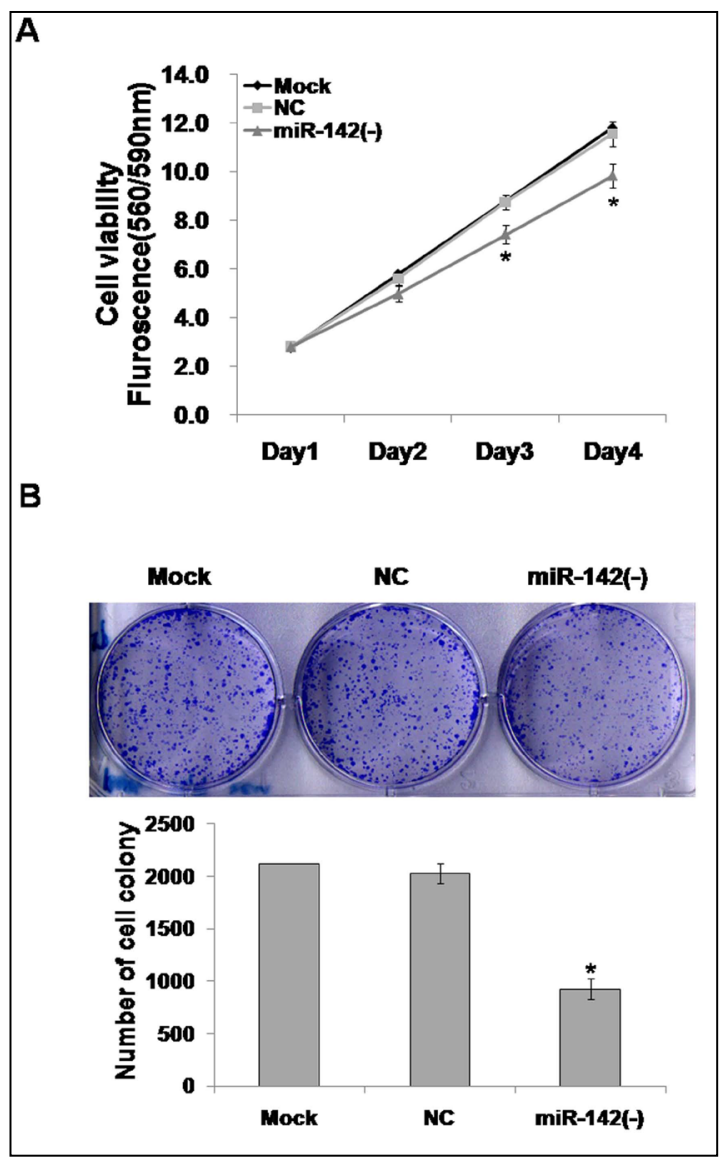

Fig. 3. Knockdown of miR-142-5p represses cell proliferation. A. Cell viability assay of ADSCs infected with pLV-miR-142-5p locker lentiviral particles was performed. B. CFU assay of ADSCs infected with pLVmiR-142-5p locker lentiviral particles was performed. The colonies were counted at 7 days after plating. Data are shown as the mean \pm S.D. of six independent experiments. ${ }^{*} \mathrm{P}<0.05$. 


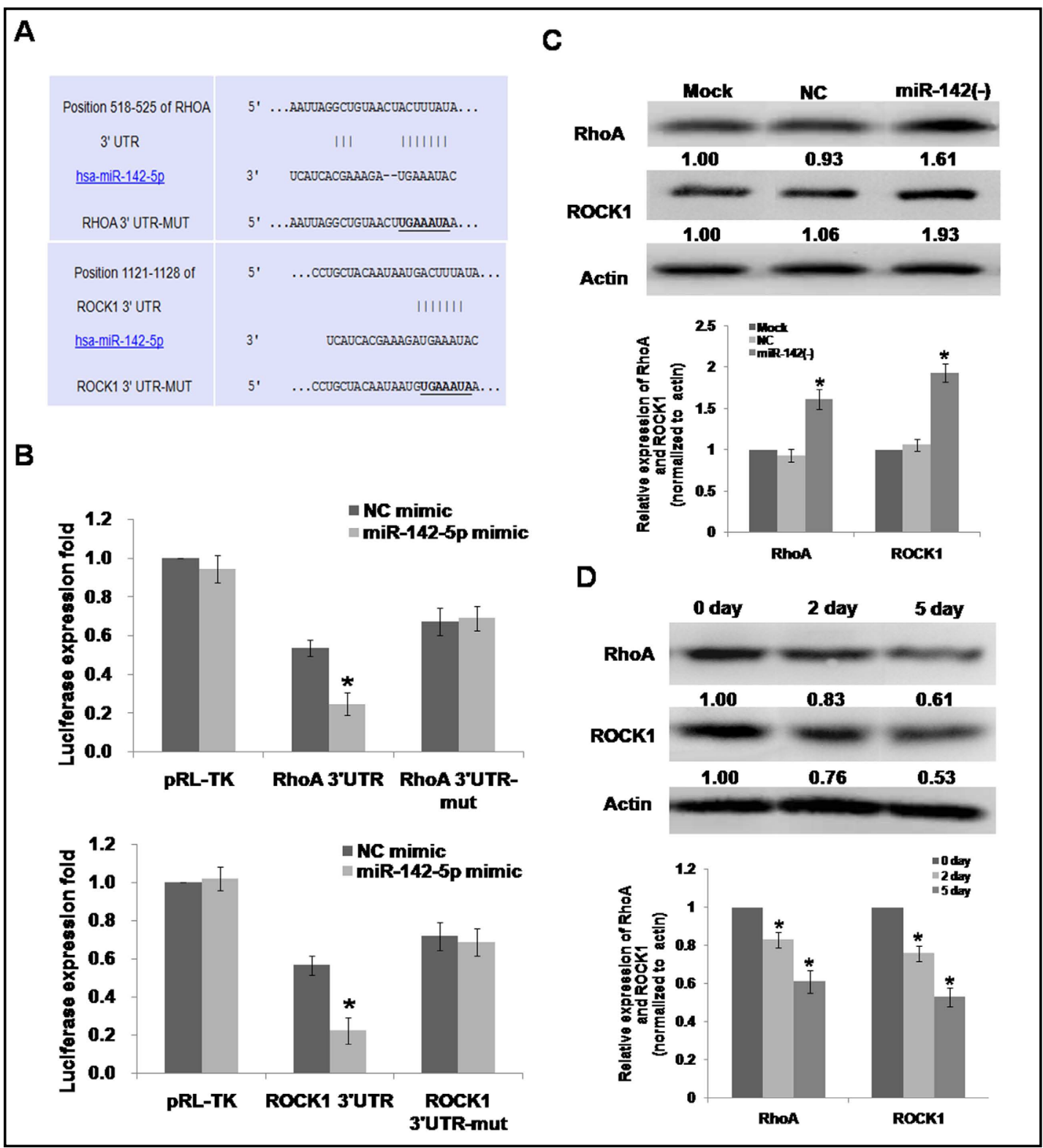

Fig. 4. MiR-142-5p directly targets RhoA and ROCK1 mRNA. A. Predicted binding sites between 3'UTR of RhoA or ROCK1 and miR-142-5p and the designed mutant sequences. B. Dual luciferase assay of relative luciferase activity of pGL3-RhoA and pGL3-RhoA-MUT in HEK 293T cells co-transfected with 50nM miR142-5p mimics or NC. Firefly luciferase activity was normalized to that of Renilla luciferase. C. Western blot analysis of RhoA and ROCK1 expression in ADSCs infected with the pLV-miR-142-5p locker lentiviral particles. D. Western blots (WB) analysis of RhoA and ROCK1 expressions in ADSCs on day 0, 2 and 5 during the differentiation induction. C\&D: the band signals of WB were analyzed by Gene tools (Syngene) and normalized to the expression of actin. Data are shown as the mean \pm S.D. of three independent experiments. $* \mathrm{p}<0.05$. 


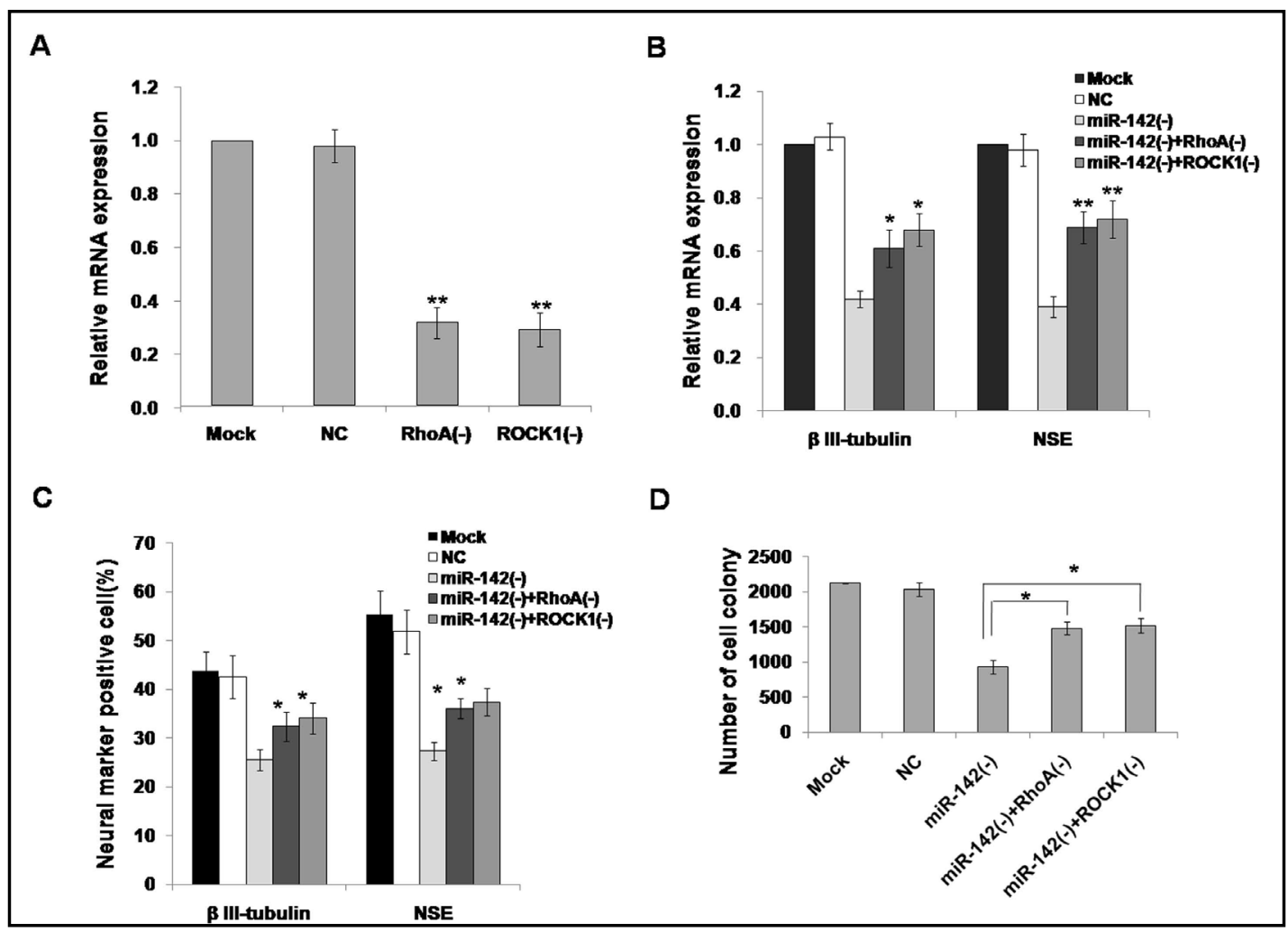

Fig. 5. MiR-142-5p enhances neuronal differentiation of ADSCs partly through RhoA/ROCK1 signaling pathway. A. qRT-PCR analysis of RhoA and ROCK1 mRNA expression in ADSCs infected with the RhoA or ROCK1 shRNA lentiviral particles. B. qRT-PCR analysis of NSE and $\beta$ III tubulin mRNA expression in infected ADSCs. C. Quantification of the proportion of neurogenic markers (NSE and $\beta$ III tubulin) positive infected ADSCs. D. Quantification of the CFU assay of infected ADSCs. B-D: ADSCs were infected with the pLV-miR142-5p locker lentiviral particles and co-infected with the pLV-miR-142-5p locker lentiviral particles and the RhoA shRNA lentiviral particles, ROCK1 shRNA lentiviral particles on day 2 after differentiation induction. Data are shown as the mean \pm S.D. of three independent experiments. ${ }^{*} \mathrm{p}<0.05,{ }^{* *} \mathrm{p}<0.01$.

\section{Discussion}

ADSCs could transdifferentiate into neuron-like cells which present multiple neuronal properties, such as synaptic transmission, action potential, secretion of dopamine and neurotrophic factors, and spontaneous postsynaptic current [11, 12]. ADSCs stimulation enhanced axon regeneration by increased GAP-43 and activating transcription factor 3 (ATF-3) expression in the spinal cord and reduced c-jun expression in the dorsal root ganglia (DRG) neurons [13]. Human ADSC extract exert a potential therapeutic action in Amyotrophic lateral sclerosis (ALS) model by reducing apoptotic cell death and recovering mutant SOD1-induced mitochondrial dysfunction [14]. ADSCs differentiated Schwann cells have the ability to improve axonal regeneration and remyelination in rat spinal cord injury $[15,16]$. Human ADSC extract ameliorated the effect of A $\beta$-induced mitochondrial oxidative stress and reduced the mitochondrial membrane potential, suggesting a therapeutic strategy to ameliorate neuronal death induced by $A \beta[17,18]$. Thus, ADSCs might have potential therapeutic applications in the treatment of neurological diseases.

Previously, many miRNAs have been reported to play a role in the proliferation and differentiation of ADSCs, such as miR-let-7e, miR-154-5p, miR-218, miR-26a, miR-27, miR31 and miR-34a [19-27]. Most recently, it was demonstrated that miR-124 is able to suppress Sp1 expression, which in turn affected the neuronal differentiation of mesenchymal stem cells [28]. miR-124 could directly target SCP1, which play a key role in the neuronal differentiation of ADSC [29]. In the present study, we found that the expression of miR-142-5p is consistently increased during the differentiation induction, together with the expression 
of neurogenic markers (NSE and $\beta$ III tubulin). These findings suggested that miR-142-5p might be involved in neuronal-like differentiation of ADSCs.

Some studies revealed that RhoA signaling pathway is involved in neuronal-like differentiation of MSCs [30-33]. And Rho-kinase (ROCK) is an important downstream effector of RhoA modulating cell fate, which is also involved in MSCs differentiation [34, 35]. Actually, the RhoA /ROCK1signaling pathway might be quite critical for neuronallike differentiation of MSCs. For example, miR-125a-3p and miR-483-5p can promote adipogenesis of ADSCs via suppressing the RhoA/ROCK1/ERK1/2 pathway [36]. MiR-335$5 \mathrm{p}$ can promote chondrogenesis of mouse MSCs by downregulating Daam1 and ROCK1 and increasing SOX9 [37]. Our preliminary study observed that miR-142-5p have the putative binding sites with 3'UTRs of RhoA and ROCK1 mRNA. Therefore, we hypothesized that miR142-5p can modulate neuronal-like differentiation of ADSCs through repressing RhoA and ROCK1. By dual luciferase assay and western blot, these targetings were verified. In addition, Knockdown of endogenous RhoA and ROCK1, partly rescued neuronal differentiation and proliferation hampered by knockdown of miR-142-5p. Thus, miR-142-5p regulating RhoA/ ROCK1 is an important axis involved in neuronal-like differentiation of ADSCs.

Previous studies have reported that the D1 dopamine receptor post-transcriptional regulation is specifically mediated by miR-142-3p in the mouse brain during postnatal development [38]. MiR-142 is overexpressed in neurons, which may contribute to the changes in dopaminergic neurotransmission by regulating SIRT1 and MAOA genes in HIV-associated neurocognitive disorders (HAND) [39, 40]. MiR-142-5p is localized to neurons and negatively regulates neuronal morphogenesisby targeting Ulk1 in porcine hemagglutinating encephalomyelitis (PHE) [41]. MiR-142-5p contributes the pathogenesis of Alzheimer's disease (AD) by triggering synaptic dysfunction associated with $A \beta 42$-mediated pathophysiology [42]. Therefore, miR-142-5p might play a critical role in neuronal process.

\section{Conclusion}

In this study, we found that miR-142-5p targets RhoA/ROCK1 signaling, which is involved in differentiation and proliferation of ADSCs for the first time. We also demonstrated that the miR-142-5p/RhoA/ROCK1 is an important signaling pathway in the differentiation. These findings provided some fundamental information for future use of ADSCs as an agent for regenerative medicine and cell therapy for neurological diseases.

\section{Acknowledgements}

This study was supported by the Natural Science Fund of China (81401006 and 81601083).

\section{Disclosure Statement}

There is no conflict of interest.

\section{References}

\footnotetext{
1 de Girolamo L, Lucarelli E, Alessandri G, Avanzini MA, Bernardo ME, Biagi E, Brini AT, D’Amico G, Fagioli F, Ferrero I, Locatelli F, Maccario R, Marazzi M, Parolini O, Pessina A, Torre ML: Italian Mesenchymal Stem Cell Group: Mesenchymal stem/stromal cells: a new "cells as drugs" paradigm. Efficacy and critical aspects in cell therapy. Curr Pharm Design 2013;19:2459-2473.

2 Stellavato A, La Noce M, Corsuto L, Pirozzi AVA, De Rosa M, Papaccio G, Schiraldi C, Tirino V: Hybrid Complexes of High and Low Molecular Weight Hyaluronans Highly Enhance HASCs Differentiation: Implication for Facial Bioremodelling. Cell Physiol Biochem 2017;44:1078-1092.
} 


\section{Cellular Physiology Cell Physiol Biochem 2018;50:2097-2107 and Biochemistry \begin{tabular}{l|l} 
DOI: $10.1159 / 000495054$ & 2018 The Author(s). Published by S. Karger AG, Basel
\end{tabular}

3 Choi JW, Shin S, Lee CY, Lee J, Seo HH, Lim S, Lee S, Kim IK, Lee HB, Kim SW, Hwang KC: Rapid Induction of Osteogenic Markers in Mesenchymal Stem Cells by Adipose-Derived Stromal Vascular Fraction Cells. Cell Physiol Biochem 2017;44:53-65.

4 Gimble JM, Katz AJ, Bunnell BA: Adipose-derived stem cells for regenerative medicine. Circ Res 2007;100:1249-1260.

5 Strem BM, Hicok KC, Zhu M, Wulur I, Alfonso Z, Schreiber RE, Fraser JK, Hedrick MH: Multipotential differentiation of adiposetissue-derived stem cells. Eio J Med 2005;54:132-141.

-6 YJ Kim, SH Hwang, HH Cho, KK Shin, YC Bae, JS Jung: MicroRNA 21 regulates the proliferation of human adipose tissue-derived mesenchymal stem cells and high-fat diet-induced obesity alters microRNA 21 expression in white adipose tissues. J Cell Physiol 2012; 227:183-193.

-7 Kim JH, Park SG, Song SY, Kim JK, Sung JH: Reactive oxygen species-responsive miR-210 regulates proliferation and migration of adipose-derived stem cells via PTPN2. Cell Death Dis 2013;4: e588.

-8 Park H, Park H, Pak HJ, Yang DY, Kim YH, Choi WJ, Park SJ, Cho JA, Lee KW: miR-34a inhibits differentiation of human adipose tissue-derived stem cells by regulating cell cycle and senescence induction.

Differentiation 2015; 90:91-100.

-9 Viswanathan SR, Daley GQ, Gregory RI: Selective blockade of microRNA processing by Lin28. Science 2008;320:97-100.

10 Hu F, Xu P, Sun B, Teng G, Xiao Z: Deep sequencing reveals complex mechanisms of microRNA regulation during retinoic acid-induced neuronal differentiation of mesenchymal stem cells. Genomics 2017;109:302311.

11 Bossio C, Mastrangelo R, Morini R, Tonna N, Coco S, Verderio C, Matteoli M, Bianco F: A simple method to generate adipose stem cell-derived neurons for screening purposes. J Mol Neurosci 2013; 51:274-81.

12 Mead B, Berry M, Logan A, Scott RA, Leadbeater W, Scheven BA: Stem cell treatment of degenerative eye disease. Stem Cell Res 2015;14:243-257.

13 Kingham PJ, Kolar MK, Novikova LN, Novikov LN, Wiberg M: Stimulating the neurotrophic and angiogenic properties of human adipose-derived stem cells enhances nerve repair. Stem Cells Dev 2014;23:741-754.

14 Jeon GS, Im W, Shim YM, Lee M, Kim MJ, Hong YH, Seong SY, Kim M, Sung JJ: Neuroprotective effect of human adipose stem cell-derived extract in amyotrophic lateral sclerosis. Neurochem Res 2016;41:913923.

15 Zaminy A, Shokrgozar MA, Sadeghi Y, Norouzian M, Heidari MH, Piryaei A: Transplantation of schwann cells differentiated from adipose stem cells improves functional recovery in rat spinal cord injury. Arch Iran Med 2013;16:533-541.

16 Liu T, Lee M, Ban JJ, Im W, Mook-Jung I, Kim M: Cytosolic Extract of Human Adipose Stem Cells Reverses the Amyloid Beta-Induced Mitochondrial Apoptosis via P53/Foxo3a Pathway. PLoS One 2017;12:e0168859.

17 Li C, Ye L, Yang L, Yu X, He Y, Chen Z, Li L, Zhang D: Rapamycin Promotes the Survival and Adipogenesis of Ischemia-Challenged Adipose Derived Stem Cells by Improving Autophagy. Cell Physiol Biochem 2017;44:1762-1774.

18 Ding C, Zou Q, Wang F, Wu H, Wang W, Li H, Huang B: HGF and BFGF Secretion by Human Adipose-Derived Stem Cells Improves Ovarian Function During Natural Aging via Activation of the SIRT1/FOX01 Signaling Pathway. Cell Physiol Biochem 2018;45(4):1316-1332.

19 Chen J, Deng S, Zhang S, Chen Z, Wu S, Cai X, Yang X, Guo B, Peng Q: The role of miRNAs in the differentiation of adipose-derived stem cells. Curr Stem Cell Res Ther 2014;9:268-279.

$>20$ Kim DY, Sung JH: Regulatory role of microRNAs in the proliferation and differentiation of adipose-derived stem cells. Histol Histopathol 2017;32:1-10.

21 Zhang WB, Zhong WJ, Wang L: A signal-amplification circuit between miR-218 and Wnt/ $\beta$-catenin signal promotes human adipose tissue-derived stem cells osteogenic differentiation. Bone 2014;58:59-66.

22 Kang T, Jones TM, Naddell C, Bacanamwo M, Calvert JW, Thompson WE, Bond VC, Chen YE, Liu D: AdiposeDerived Stem Cells Induce Angiogenesis via Microvesicle Transport of miRNA-31. Stem Cells Transl Med 2016;5:440-450.

-23 Kang T, Lu W, Xu W, Anderson L, Bacanamwo M, Thompson W, Chen YE, Liu D: MiR-27 targets prohibitin and impairs adipocyte differentiation and mitochondrial function in human adipose-derived stem cells. J Biol Chem 2013;288:34394-34402. 


\section{Cellular Physiology Cell Physiol Biochem 2018;50:2097-2107 and Biochemistry \begin{tabular}{l|l|l} 
DOI: 10.1159/000495054 & (c) 2018 The Author(s). Published by S. Karger AG, Basel \\
www.karger.com/cpb
\end{tabular}}

Yang et al.: miR-142-5p Targeting RhoA/ROCK1 in ADSCs

-24 Park H, Park H, Pak HJ, Yang DY, Kim YH, Choi WJ, Park SJ, Cho JA, Lee KW: miR-34a inhibits differentiation of human adipose tissue-derived stem cells by regulating cell cycle and senescence induction. Differentiation 2015;90:91-100.

25 Li J, Hu C, Han L, Liu L, Jing W, Tang W, Tian W, Long J: MiR-154-5p regulates osteogenic differentiation of adipose-derived mesenchymal stem cells under tensile stress through the Wnt/PCP pathway by targeting Wnt11. Bone 2015;78:130-141.

26 Ventayol M, Viñas JL, Sola A, Jung M, Brüne B, Pi F, Mastora C, Hotter G: miRNA let-7e targeting MMP9 is involved in adipose-derived stem cell differentiation toward epithelia. Cell Death Dis 2014;5:e1048.

-27 Wang Z, Xie Q, Yu Z, Zhou H, Huang Y, Bi X, Wang Y, Shi W, Sun H, Gu P, Fan X: A regulatory loop containing miR-26a, GSK3 $\beta$ and C/EBP $\alpha$ regulates the osteogenesis of human adipose-derived mesenchymal stem cells. Sci Rep 2015;5:15280.

-28 Mondanizadeh M1, Arefian E, Mosayebi G, Saidijam M, Khansarinejad B, Hashemi SM: MicroRNA-124 regulates neuronal differentiation of mesenchymal stem cells by targeting Sp1 mRNA. J Cell Biochem 2015;116:943-953.

29 Shi F, Yang Y, Wang T, Kouadir M, Zhao D, Hu S: Cellular prion protein promotes neuronal differentiation of adipose-derived stem cells by upregulating miRNA-124. J Mol Neurosci 2016;59:48-55.

30 Gu H, Yu SP, Gutekunst CA, Gross RE, Wei L: Inhibition of the Rho signaling pathway improves neurite outgrowth and neuronal differentiation of mouse neural stem cells. Int J Physiol Pathophysiol Pharmacol 2013;5:11-20.

31 Matsumoto Y, Inden M, Tamura A, Hatano R, Tsukita S, Asano S: Ezrin mediates neuritogenesis via downregulation of RhoA activity in cultured cortical neurons. PLoS One 2014;9:e105435.

32 Kouchi Z, Igarashi T, Shibayama N, Inanobe S, Sakurai K, Yamaguchi H, Fukuda T, Yanagi S, Nakamura Y, Fukami K: Phospholipase Cdelta3 regulates RhoA/Rho kinase signaling and neurite outgrowth. J Biol Chem 2011;286:8459-8471.

-33 Jeon CY, Kim HJ, Morii H, Mori N, Settleman J, Lee JY, Kim J, Kim SC, Park JB: Neurite outgrowth from PC12 cells by basic fibroblast growth factor (bFGF) is mediated by RhoA inactivation through p190RhoGAP and ARAP3. J Cell Physiol 2010;224:786-794.

-34 Ferrari A, Cecchini M, Serresi M, Faraci P, Pisignano D, Beltram F: Neuronal polarity selection by topography-induced focal adhesion control. Biomaterials 2010;31:4682-4694.

35 Gu X, Meng S, Liu S, Jia C, Fang Y, Li S, Fu C, Song Q, Lin L, Wang X: miR-124 represses ROCK1 expression to promote neurite elongation through activation of the PI3K/Akt signal pathway. J Mol Neurosci 2014;52:156-165.

-36 Chen K, He H, Xie Y, Zhao L, Zhao S, Wan X, Yang W, Mo Z: miR-125a-3p and miR-483-5p promote adipogenesis via suppressing the RhoA/ROCK1/ERK1/2 pathway in multiple symmetric lipomatosis. SciRep 2015; 5:11909.

-37 Lin X, Wu L, Zhang Z, Yang R, Guan Q Hou X, Wu Q: MiR-335-5p promotes chondrogenesis in mouse mesenchymal stem cells and is regulated through two positive feedback loops. J Bone Miner Res 2014;29:1575-1585.

-38 Tobón KE, Chang D, Kuzhikandathil EV: MicroRNA 142-3p mediates post-transcriptional regulation of D1 dopamine receptor expression. PLoS One 2012;7:e49288.

39 Chaudhuri AD, Yelamanchili SV, Fox HS: MicroRNA-142 reduces monoamine oxidase A expression and activity in neuronal cells by downregulating SIRT1. PLoS One 2013;8(11):e79579.

40 Thapa I, Fox HS, Bastola D: Coexpression network analysis of miRNA-142 overexpression in neuronal cells. Biomed Res Int 2015;2015:921517.

41 Li Z, Lan Y, Zhao K, Lv X, Ding N, Lu H, Zhang J, Yue H, Shi J, Song D, Gao F, He W: miR-142-5p disrupts neuronal morphogenesis underlying porcine hemagglutinating encephalomyelitis virus infection by targeting Ulk1. Front Cell Infect Microbiol 2017;7:155.

-42 Song J, Kim YK: Identification of the role of miR-142-5p in Alzheimer's disease by comparative bioinformatics and cellular Analysis. Front Mol Neurosci 2017;10:227. 